\title{
Vagal Tone Biofeedback: Respiratory and Non-respiratory Mediated Modulations of Vagal tone Challenged by Cold Pressor Test
}

\author{
Dorey Glenn, BSc*, Blaine Ditto, $\mathrm{PhD}$
}

\begin{abstract}
Deficient parasympathetic activity to the heart has been hypothesized to underlie some forms of cardiovascular disease. Consequently, this experiment attempted to induce nonrespiratory mediated increases in cardiac parasympathetic activity. Thirty-nine subjects were asked to increase their vagal tone using biofeedback, paced breathing, biofeedback plus paced breathing, or quiet sitting. The cold pressor test was used to examine the relative efficacy of vagal tone increase to mitigate cardiovascular reactivity. Repeated measures ANOVAs and t-tests revealed significant increases in vagal tone for the paced breathing, and biofeedback plus paced breathing groups, relative to controls. However, the quality of these increases could not be tested using the cold pressor test because the test failed to produce homogeneous cardiac reactivity.
\end{abstract}

\section{INTRODUCTION}

Heart disease and stress-related disorders are among the leading causes of death in the United States and Canada (American Heart Association, 2003; Statistics Canada, 2000). Deficient parasympathetic activity to the heart has been hypothesized to underlie some forms of cardiovascular disease, including cardiac arrhythmias (Eckberg et al., 1971 and 1980). Cardiac parasympathetic activity, or the amount of bioelectrical flow through the vagus nerve (i.e. vagal tone), provides homeostatic and inhibitory influences on the heart (Porges, 1993). Recently, indices of parasympathetic tone to the heart have been shown to reflect overall

\footnotetext{
* To whom correspondence should be addressed: Dorey Glenn, 3941 Rivard St., Montreal, Quebec Canada, H2L 4H8. Phone number: (514) 843 7625. E-mail: Dag1111@yahoo.com
}

cardiopulmonary health (23), and thus, methods to increase vagal activity have become of clinical interest. Various psychophysiological, behavioral, and pharmacological methods-including biofeedback-have been devised to increase cardiac vagal activity $(22,13$, 26). Vagal tone is most commonly measured by analyzing the rhythmical variations in heart rate period due to the respiratory cycle, generally referred to as the respiratory sinus arrhythmia (RSA) (17).

Previous studies have been able to significantly increase vagal activity $(26,28)$, but have failed to test these increases with a stressor. Due to the absence of such testing, it is not known whether previous experimental increases in vagal activity are able to mitigate physiological arousal. Acute stressors, such as the cold pressor test, can be used to test the efficacy of 
training methods and the quality of the resultant increases in vagal tone (30). In addition, past attempts to experimentally increase vagal tone have failed to control for respiratory influences on vagal activity (26). Changes in the respiratory pattern have a profound influence on cardiac variability, and thus readily influence measurements of cardiac parasympathetic activity. The following investigation attempted to use a biofeedback paradigm to isolate non-respiratory increases in vagal tone, and tested the resiliency of these increases with the cold pressor test. Successful increases in vagal tone will not only add support to the role of biofeedback and respiratory control in the treatment of some forms of heart disease, but will shed light on a non-respiratory alternative to vagal tone increases.

\section{Background}

The American Heart Association (AHA) estimates that cardiovascular disease costs the United States 351.2 billion dollars in indirect and direct economic losses per year (American Heart Association, 2003). Furthermore, the AHA estimates that cardiac arrhythmias cost Medicare approximately 2.1 billion dollars in 2000 . Arrhythmias were mentioned as both the cause of death of 37,646 people, as well as an underlying or contributing cause of death in 461,000 persons in 2002. Due to an increasing proportion of elderly people in both the American and Canadian populations, rates of myocardial infarct and cardiovascular related hospitalizations are expected to rise continuously in the next 20 years (Statistics Canada, 2000). Although cardiovascular disease is a largely preventable pathology, its frequent occurrence has spurned much pathophysiological research, and thus has helped to reveal much of the physiology underlying both normal and abnormal cardiovascular function (18).

The autonomic nervous system has been functionally and anatomically divided into the parasympathetic and sympathetic divisions. The former division is responsible for homeostatic and regenerative functions, and the latter division supports behavioral responses to threat and the mobilization of metabolic resources. The vagus nerve, part of the parasympathetic division, is responsible for much of the neural control of the internal viscera; however, its influence on the heart and lungs is of most concern in the present discussion. Increased parasympathetic activity to the heart decreases heart rate, and in conjunction with respiratory rate and tidal volume, determines respiratory sinus arrhythmia (RSA).

Respiratory sinus arrhythmia is the close occurrence of cyclical fluctuations in heart rate to the respiratory tides. As early as 1733, Hales observed RSA by noticing that changes in pulse were related in a regular manner to the respiratory pattern in the horse. In 1910, Hering had identified the vagus nerve as being responsible for the lowering of heart rate during respiration (23). Since then, computation of respiratory sinus arrhythmias has increasingly been used as a reliable, selective, and non-invasive measure of vagal activity to the heart (5).

RSA is calculated using the slight variation between heartbeat intervals. The presumed purpose of RSA is to maximize oxygen transport during inhalation, and carbon dioxide diffusion during exhalation (22). This physiological mechanism is controlled by the extent of neural activity through the vagus nerve, and can thus serve as a non-invasive measure of parasympathetic tone to the heart (22). Although many factors may produce rhythmical fluctuation in heart rate, the highfrequency component of heart rate variation is principally mediated by the vagus nerve (12). For example: pharmacological parasympathetic blockade (by atropine), but not sympathetic blockade, abolishes respiratory sinus arrhythmia, implicating the vagus nerve as the dominant source of RSA $(22,13)$. Another important factor influencing vagal activity is respiration, which will be discussed in context below.

Parasympathetic tone serves a protective function for the heart, whereby increased parasympathetic tone produces decreased heart rate and increased ability to mitigate sympathetic arousal (23). Porges explains: "Heart rate variability is a marker of the efficiency of neural feedback mechanisms and may index health status or the individual's capacity to organize physiological resources to respond appropriately. Thus, the better the 'organized' physiologic variability, the greater the range of behavior...[and] flexibility in response to environmental demands [or disease states]." In other words, heart rate variability characterizes efficient and sensitive neural control, and thus is a predictor of healthy resilience to stress. Therefore, states of attenuated vagal influence on the heart, manifesting as decreased heart rate variability, are "paralleled by reduced behavioral flexibility in response to environmental demands," and increased susceptibility to a variety a cardiovascular pathologies (23).

Both human and animal studies have revealed a clear association between reduced parasympathetic activity and cardiovascular dysfunction $(9,10)$. An analysis of the heart rate variability of 808 acute myocardial infarction survivors revealed that heart rate variability was a significant predictor of long-term survival (18). The authors suggest that decreased vagal tone, responsible for decreased heart rate variability, may predispose the heart to ventricular fibrillation. 
Likewise, in animals with experimental myocardial infarcts, decreased vagal tone and reduced heart rate variability predisposed the animals to ventricular fibrillation (20).

Furthermore, evidence revealing high correlations between decreased RSA and arteriosclerotic severity (14), and RSA amplitude and the sensitivity of the baroreceptor reflex provide further support of the importance of parasympathetic activity in cardiac health in normal populations (26). Negative correlations between RSA amplitude and blood pressure were found in hypertensive populations (11).

Despite the importance of vagal activity, relatively few non-pharmacological methods to increase vagal tone have been applied at the clinical level $(19,28)$. Among a variety of possible approaches, including autogenic training, progressive relaxation, and transcendental meditation, biofeedback has made important contributions to the field of behavioral modification and psychophysiology (21). Biofeedback is the closure of a loop connecting a physiological variable to a willful individual able to, at some level, control that variable. Although both an awareness model and an operant conditioning model have been proposed as possible mechanisms underlying biofeedback control (6), etiology will not be of concern in this experiment. Biofeedback attempts to mimic the numerous regulatory feedback loops underlying the homeostatic balance essential for life (3). Historically, biofeedback has been used with varying success for a number of clinical pathologies including: hypertension, cardiac arrhythmias, migraine headaches, Raynaud's syndrome, tension headaches, test anxiety, seizure activity, stomach acidity, and incontinence (21).

Many studies have been conducted exploring the efficacy of biofeedback paradigms in manipulating heart rate (25), and to a lesser extent, heart rate variability. One study by Reyes et al. (26) attempted to differentially increase RSA magnitude using variations in biofeedback procedures. Forty subjects were randomly assigned to four conditions: RSA biofeedback without respiratory instructions, RSA biofeedback with respiratory instructions, respiratory biofeedback or, d) respiratory instruction only. Respiratory instructions consisted of slow and deep breathing. Although all groups produced significant increases in RSA from baseline, the respiratory biofeedback group produced the fastest increases in RSA amplitude, and the RSA biofeedback group without respiratory instructions produced the slowest. Between the remaining conditions, respiratory instructions produced faster increases in RSA than did RSA biofeedback with respiratory instructions.

The primacy of respiratory instructions to increase RSA magnitude is not surprising considering that low frequency and high amplitude respiratory patterns induce increases in RSA. However, why should the addition of biofeedback retard RSA increases despite both groups having been given respiratory instructions, as indicated by the latter finding? The authors attribute this peculiar result to "the nature of the target used in each trial." The respiratory instructions group directly manipulated the variable used to control RSArespiration. The RSA biofeedback group with respiratory instructions attempted to control the variable itself-RSA. The authors view this evidence as reason to focus on: "the control strategies [respiration] [rather] than directly on the physiological variable to be controlled [vagal tone]."

This conclusion may be premature. Increases in RSA were not subjected to a real world "test" or validation; namely, an acute stressor. Thus, one cannot be sure of the quality of these increases. Perhaps respiratory procedures only transiently induce increased RSA, albeit faster. Can these increases withstand or mitigate reactivity to an acute stressor? Does the operant conditioning of biofeedback create a more sustainable form of RSA increase than respiratory instructions alone? Respiratory instructions are cognitive tools subject to degradation and non-use in stressful situations. However, biofeedback learning can produce a pattern or conditioned response to a stressor. In other words, increases in vagal tone must be of good quality, and not necessarily of good quantity. Sakakibara et al. (28) emphasize that "respiratory parameters need to be controlled when assessing the cardiac parasympathetic tone by the magnitude of the respiratory sinus arrhythmia."

The following experiment attempted to separate vagal tone increases due to respiratory manipulations from those increases due to direct, biofeedback-induced, increases in vagal tone. Evidence supporting this approach was found by Sakakibara et al. (1994). During autogenic relaxation, an increase in vagal tone was found even when subjects' respiratory rate and volume were controlled by means of a pneumogram and strain gauge. This showed that vagal tone could be manipulated even when respiration is controlled. Both the mechanism for producing such increases and whether such an effect can be reproduced remains unclear. Nonetheless, Sakakibara et al. (28) hypothesize that enhanced cardiac parasympathetic tone may be one mediator of the relaxation response, as coined and popularized by Benson (4).

Vagal increases due to respiratory instruction or respiratory biofeedback, albeit more rapid, may be as topical and transient as the respiratory cycle itself, and thus susceptible to the vagaries of modern life. It is yet unclear if biofeedback procedures can tap into a 
respiratory-contamination-free aspect of vagal tone. By pacing the respiratory rate at a constant rhythm, while administering vagal tone biofeedback, this experiment attempted to produce increases in cardiac parasympathetic tone that were free of respiratory influence.

The cold pressor test was used in conjunction with biofeedback to act as a source of both sympathetic arousal and parasympathetic withdrawal; in other words, as a model for an acute stressor. Past biofeedback research has produced significant increases and mild decreases in heart rate (31). Both effects have been shown to persist through the cold pressor test. Furthermore, placebo effects, or changes in heart rate and subjective pain ratings due simply to the belief in heart rate change, do not account for reported subjective pain ratings or heart rate changes (24). In summary, biofeedback training has been shown to effectively produce an increased heart rate that persists through an acute stressor, and that cannot be accounted for by placebo. Whether the same is true for vagal tone is yet unclear.

The present study attempted to increase vagal tone by using biofeedback, while maintaining constant respiration. The durability of these increases was tested using the cold pressor test.

\section{METHODS}

\section{Participants}

Subjects were 13 male and 26 female undergraduates (mean age $=21 \pm 2.3$ ) from McGill University solicited using ads posted around campus. Strenuous physical activity and the use of over-the-counter medication, caffeine, and nicotine were prohibited for four hours prior to experimentation. Participants were compensated $\$ 10$ for their time.

\section{Apparatus}

A Delta Biometrics Inc. vagal tone Monitor II was used to compute heart rate and vagal tone data. Subjects were comfortably seated approximately 2 feet from the feedback display in a padded chair. After alcohol swabbing, two hypoallergenic electrodes were placed on the lower rib cage, and a third ground electrode was placed on the ankle. The electrocardiogram obtained from these electrodes was amplified using a Grass polygraph and processed, in turn, by the Vagal Tone Monitor II, which quantifies beat-to-beat heart rate variability in the 0.15 to $0.40 \mathrm{~Hz}$ frequency band (i.e. respiratory sinus arrhythmia).

A 2-liter plastic container was filled with 2 trays of ice cubes and 1 liter of cold water at a temperature of 4 degrees Celsius for the cold pressor test. An electronic thermometer was used to insure a stable and adequate temperature. A wire mesh was placed diagonally in the tub to separate the ice from contact with the subject's hand.

In place of a metronome, a computer program simulating a $0.25 \mathrm{~Hz}$ sin wave was displayed approximately 2 feet from subjects on a laptop computer screen. Participants were initially trained to pace their respiration to an " $\mathrm{X}$ " moving along the sin wave.

\section{Procedure}

To assess the differential efficacy of vagal tone biofeedback and controlled breathing to increase RSA at rest and during the cold pressor test, subjects were randomly assigned to one of four experimental conditions. The vagal tone biofeedback condition (B), vagal tone biofeedback and paced breathing condition $(\mathrm{PB}+\mathrm{B})$, paced breathing only $(\mathrm{PB})$, and sitting quietly (C). All groups practiced their respective manipulations for two 15-minute sessions.

Following informed consent and a brief introduction, all subjects sat quietly for 5 minutes in order to reach a baseline measure of vagal tone (see Table 1 for an outline of the experimental session). An initial 1 minute cold pressor test was administered to assess baseline vagal reactivity. Five minutes of quiet sitting followed to allow for vagal tone recovery. Subsequently, each experimental group had 2 sessions of 15 minutes to practice their respective manipulation. Between sessions, a 3 minute break was provided. After the second trial, all subjects were asked to retake the cold pressor test. Subjects sat quietly for another 5 minutes to allow for the final vagal tone reading. Subjects in the paced breathing group were asked to pace their breath with a $0.25 \mathrm{~Hz}$ moving sign wave.

Subjects in the biofeedback condition were asked to increase the displayed (vagal tone) readout. The true nature of the number was not revealed, although subjects were told that relaxation would help make the number rise. The biofeedback and paced-breathing group were both given the above instructions. This group was instructed to first feel comfortable breathing consistently with the computer program, and then move towards increasing the feedback display. Subjects in the control group were asked to sit quietly.

Table 1: Outline of Experimental Session

\section{Procedure}

Baseline- 5 min

Baseline cold pressor test- 60 sec

Rest 1-5 min

Practice Session 1- 15 min

Break- 1 min

Practice Session 2- 15 min

Cold pressor test 2 - $60 \mathrm{sec}$

Rest 2 - 5 min 
As suggested by Reyes et al. (26), subjects attempting vagal tone increases using biofeedback (groups $B$ and $\mathrm{PB}+\mathrm{B}$ ) were informed that the feedback is normally quite variable, and that they should not be discouraged by seemingly incongruous feedback. Rather, they should focus on the general trend of the feedback readout.

Vagal tone measurements were taken in 5 second increments throughout the entire experimental session. Mean values of vagal tone were subsequently computed for each portion of the experimental procedure. Data files were transferred from the Vagal Tone Monitor II and analyzed using Microsoft Excel spreadsheets. Before debriefing, participants were asked to fill out a questionnaire describing their subjective experiences of each portion of the experiment, and their strategies for achieving the goal of their respective treatments. They were also asked to rate their subjective pain during the second cold pressor, and whether they found the second cold pressor to be more or less painful than the first.

\section{Statistical Analysis}

Repeated measures ANOVAs and t-tests were used to assess differences within and between experimental conditions. The time variable (within subject), treatment variable (between subject), and interactions were calculated using SYSTAT software.

\section{Expected Results}

It was hypothesized that the paced breathing plus biofeedback $(\mathrm{PB}+\mathrm{B})$ and biofeedback $(\mathrm{B})$ conditions would produce the greatest increases in vagal tone. The control group (C) should show no increases, and the paced breathing group $(\mathrm{PB})$ should show only moderate increases in vagal tone. Furthermore, although all the experimental groups should show less cardiac reactivity to the second cold pressor test, the paced breathing plus biofeedback ( $\mathrm{PB}+\mathrm{B})$ group should demonstrate the greatest ability to mitigate the physiological arousal of this stressor.

\section{RESULTS}

The effects of the four conditions on seated, nonstress values of RSA, were examined using a 4 (group) X 3 (baseline, session 1, session 2) analysis of variance (ANOVA). This ANOVA revealed a significant interaction between condition and time $(\mathrm{F}(6,70)=2.43$, $\mathrm{p}=0.044)$. All three treatment groups, $(\mathrm{PB}, \mathrm{B}, \mathrm{PB}+\mathrm{B})$ produced increases in vagal tone compared to controls. The paced breathing $(\mathrm{t}(17)=1.97, \mathrm{p}=0.034)$ and paced breathing plus biofeedback ( $(18)=2.63, \mathrm{p}=0.032$ ) groups produced significant increases during the second practice session compared to controls. The biofeedback condition produced increases in vagal tone that approached significance $(\mathrm{t}(18)=1.1, \mathrm{p}=0.143)$. T-tests revealed no significant differences between groups at baseline, but significant differences during the second practice session, indicating that the vagal increases were due to the experimental manipulations and not time (Figure 1 and $1 b$ ).

As expected, subjects in the four groups did not differ significantly in baseline vagal tone, or reactivity to the first cold pressor test. However, to ensure that all increases were not an artifact of slightly, though nonsignificant, increased baseline vagal tone, as was the case for the biofeedback plus paced breathing group, session 2 scores were subtracted from baseline scores and then re-subjected to analysis of variance and t-tests. No differences were observed in the adjusted results, supporting the reliability of the vagal increases.

To address the possibility that the experimental increases were of differing quality, first change scores were calculated. Differences between the first cold

Figure 1 Modulation of vagal tone during experimental session ( \pm standard error)

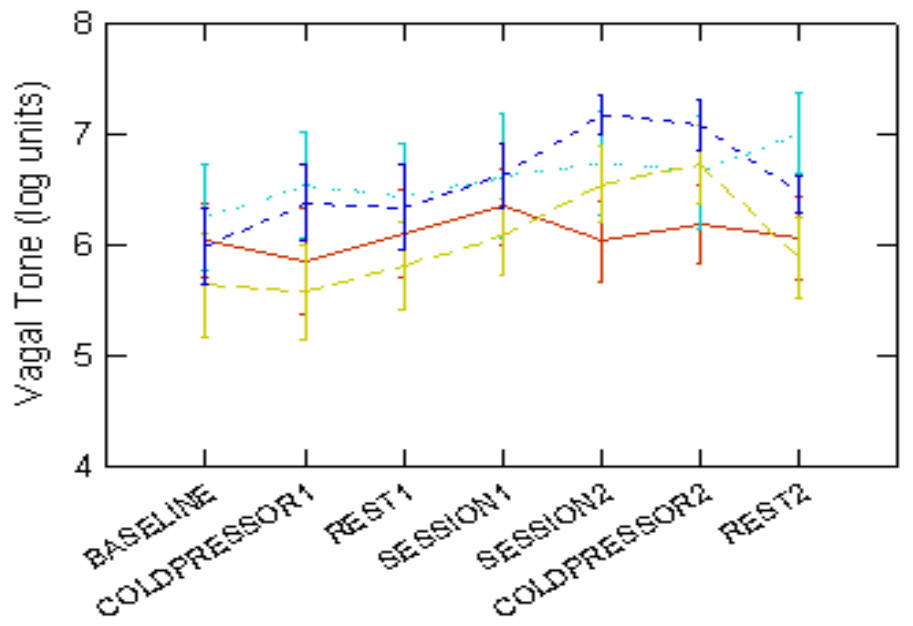

Experimental Session

\section{CONDITION}

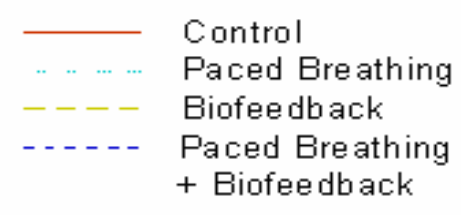


Figure 1b Modulation of vagal tone during experimental sessions. * $(\mathrm{t}(17)=1.97, \mathrm{p}=0.034) * *(\mathrm{t}(18)=2.63, \mathrm{p}=0.032)( \pm$ standard error)

Control

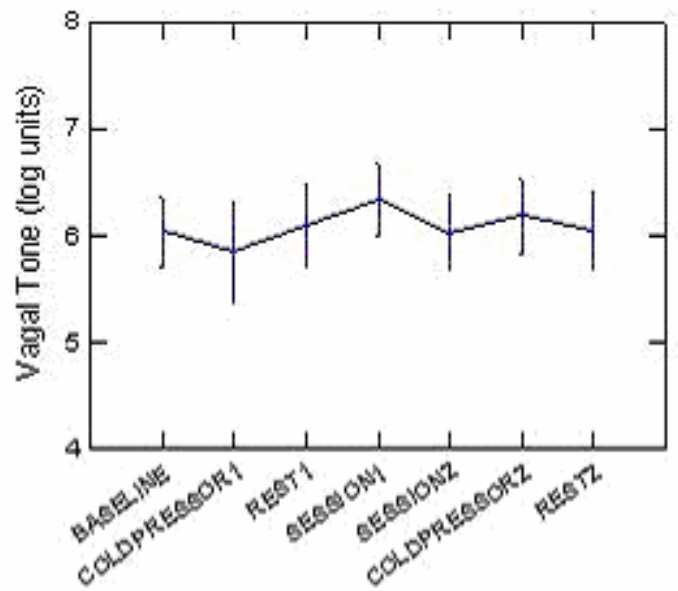

Biofeedback

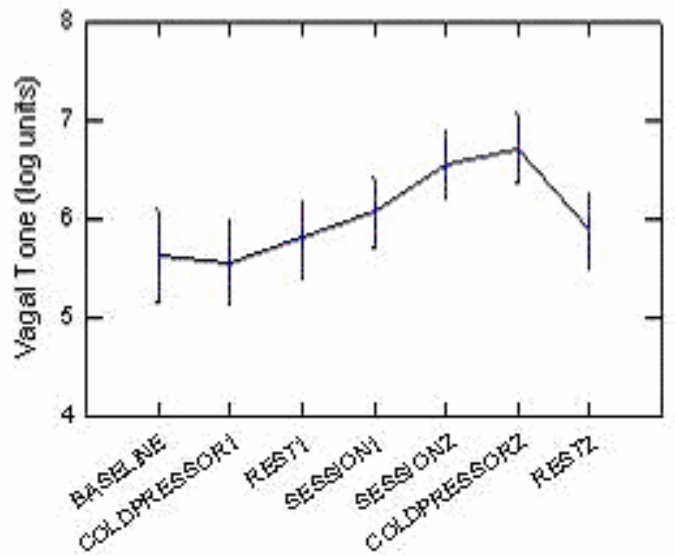

pressor and baseline, and the second cold pressor test and session 2 were analyzed using repeated measures a 4 (group) X 2 (cold pressor) ANOVA. There were no significant interactions between condition and cold pressor reactivity $(\mathrm{F}(3,35)=0.878, \mathrm{p}=0.46)$. The failure to find evidence to support differences in vagal quality is thought to reflect the insufficiency of the cold pressor to produce homogeneous physiological stress in subjects.

Similar analyses were performed on heart rate data. No significant interactions were found. However, as heart rate is not of primary concern to the present hypotheses, it will not be discussed further.

\section{DISCUSSION}

This investigation attempted to not only study whether biofeedback (B) and paced breathing (PB) could differentially increase vagal tone, but whether the
Paced Breathing

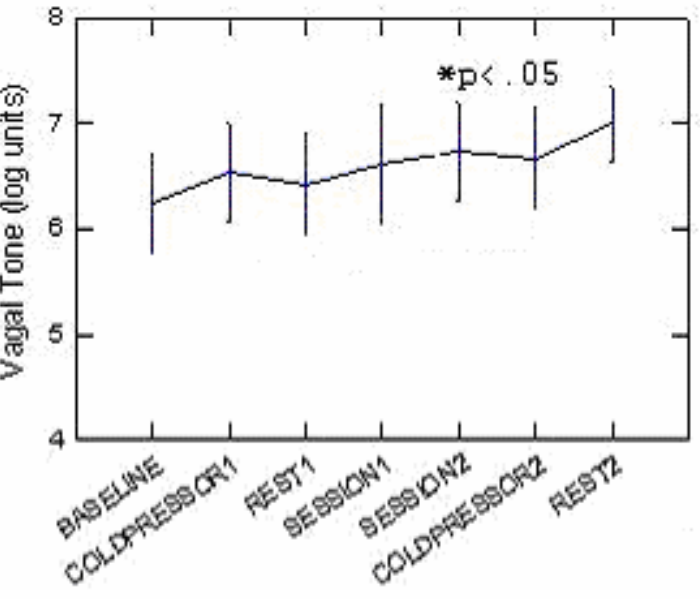

Pac. Bre. + Bio

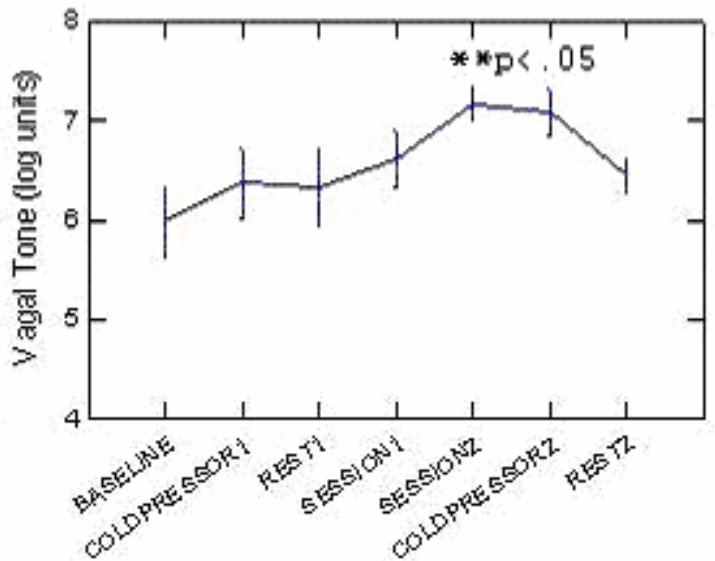

combination $(\mathrm{PB}+\mathrm{B})$ could induce non-respiratory mediated increases in vagal tone. Furthermore, it was hoped that increases in vagal activity in the $\mathrm{PB}+\mathrm{B}$ condition could better mitigate the stressful effects of the cold pressor test. Due to the failure of the cold pressor test to produce homogeneous physiological reactivity, only the prior question could be addressed.

\section{Experimental Groups}

Significant increases in vagal tone were observed in the paced breathing and paced breathing plus biofeedback conditions. These results replicate those of Reyes et al. (1992). As mentioned earlier, such increases in vagal tone are not surprising because increases in respiratory depth and frequency are known to boost vagal tone, and thus RSA.

Whether the significant increases in the paced breathing plus biofeedback group can be attributed to 
the effects of the paced respiration, or to the production of non-respiratory mediated vagal tone is not overtly clear. It is possible that respiratory-based increases in vagal tone, as demonstrated by the paced breathing group, may have overshadowed any biofeedback effects. In this view, the observed vagal increases must be attributed to respiration. On the other hand, it is possible that the biofeedback protocol may have reinforced any respiratory effects, through its feedback conditioning, thereby producing more durable vagal tone. Subjecting these increases to a reliable stressor, such as timed serial subtraction or speech tasks, would have been helpful in determining the relative efficacies of respiration and biofeedback in the production of good quality vagal tone. It is unclear if such increases resemble those found by Sakakibara et al. (28) during autogenic relaxation.

Although the biofeedback condition alone did not produce significant increase in vagal activity, increases were in the predicted direction. A number of factors may account for the demonstrated results.

Firstly, among all three experimental groups, subjects in the biofeedback condition had the most difficult task. They had to search for control strategies for the feedback by trial and error. It is not clear exactly how long such a search should take, although our data suggest that subjects improved from session 1 to session 2. In fact, as shown in Figures 2 and 3, this group produced the greatest-albeit non-significant-differences in vagal tone between the two practice sessions.

Secondly, in contrast to paced breathing, the practice session may not have been of sufficient duration to maximize the biofeedback effects. The data reveals this group to have the largest, though non-significant, difference between session 1 and session 2, indicating a longer learning period (Figure 2 and 3). Previous biofeedback research has used variable practice lengths and multiple practice sessions $(19,26,28)$. Further investigation is needed to accurately gauge the optimal duration and frequency of practice sessions. Consideration, however, should be given to the clinical, financial, and logistical factors critical for the applied relevance of such research.

Thirdly, this group's feedback was of the visual type and highly variable, resulting in a lengthy period of adjustment. Subjects in the biofeedback group were required to keep their eyes open, and focus on a display. Such focused attention may have prevented them from fully relaxing, or concentrating on finding an adequate control strategy. The high variability of the output may have similarly hindered subjects' attempts at relaxation and control. It was felt that greater feedback data, in the form of 5 second intervals, was necessary to assist the subjects in the search for, and conditioning of, an accurate control strategy. More work must be done to investigate the trade-off between variability and feedback accuracy during biofeedback.

Similar issues may have also been present for subjects in the paced breathing plus biofeedback group. Their task, besides balancing the two required tasks, was to produce increases in vagal tone irrespective of respiratory manipulation. The experimental setup, however, may not have been ideal to induce such increases. Firstly, vagal increases due to the paced breathing may have become visible through the biofeedback display. Hence, subjects may have been reinforcing their respiratory maneuvers rather than producing increases from novel, non-respiratory

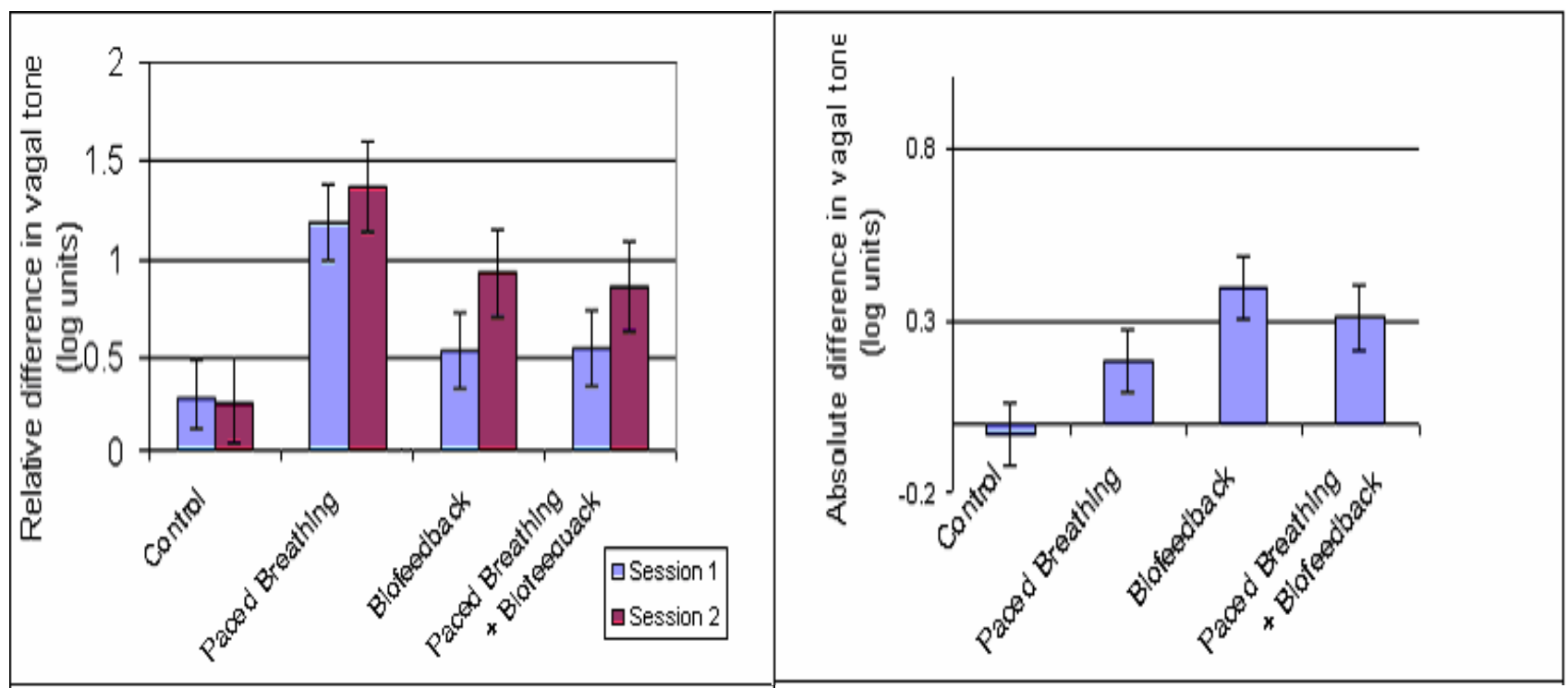

Figures 2 Relative differences in vagal tone between session 1 and session 2 ( \pm standard error)

Figure 3 Absolute differences in vagal tone between session 1 and session 2 ( \pm standard error) 
related, control strategies. The use of a pneumogram would have been useful to ensure that subjects did not inadvertently alter their respiration in an attempt to increase the feedback display in all conditions.

Secondly, one cannot be sure of the extent and proportion of subjects' directed attention towards the biofeedback display. Although subjects were instructed to first master the paced breathing and then move on to the display, they may have found respiration a more "pleasant" and rewarding strategy, in which case their results would reflect the paced breathing group. Likewise, subjects may have focused only on the biofeedback task. A more harmonious integration of the paced breathing and biofeedback tasks, as well as the addition of a check to ensure compliance, would be of great value in future attempts to produce valid and reliable non-respiratory mediated increases in vagal tone. Assigning the tasks to two separate sensory modalities, by using auditory rather than visual feedback, for example, might ease the integration of the tasks.

\section{Cold Pressor Test}

As a result of the failure of the cold pressor test to evoke consistent decreases in vagal tone, and of the resulting fractioned subject group being too small for analysis, the quality of vagal increases could not be tested.

The subject population did not react homogenously to the cold pressor test. Only 17\% $(\mathrm{n}=7)$ reacted to the cold pressor in the predicted fashion, with decreases in vagal tone to both cold pressors. However, $33 \%(n=13)$ of the subjects reacted with increases in vagal tone to both cold pressors. Although not verified, these increases may have been due to hemodynamic vasoconstriction, blood pressure increases, and subsequent baroreceptor medicated bradychardia. This hemodynamic response may have overshadowed any parasympathetic withdrawal, rendering the majority of the subjects inadequate for analysis. The remaining $50 \%$ of subjects $(n=19)$ did not show consistent reactions in either direction to the cold pressor test. Their vagal activity was either neutralized by a possible hemodynamic response, or was altogether absent.

Additionally, the participants in the present study were young and healthy. Their cardiac and hemodynamic reactivity to the cold pressor stimulation exemplified a healthy "capacity to organize physiological resources" to respond to environmental demands (23). Experimentally speaking, what one gains in fine-tuned and efficient neural feedback by using such subjects, one loses in the homogeneity of stress reactivity. Likewise, it is unhealthy and comparatively older individuals to whom increased vagal tone is an asset. Thus, these results cannot necessarily be generalized to the population to which this research is applicable.

Although a commonly used stressor, both increases and decreases in vagal tone have been observed in response to the cold pressor test. Both water temperature and body part (i.e. forehead, foot, hand) that was subjected to stimulation, are important factors influencing cardiac and hemodynamic responses to stimulation (30). Since all participants in the present study were able to withstand the full 1 minute test, perhaps an increased stimulation period, or decreased water temperature, would have produced more pain and thus more consistent decreases in vagal tone. It is unclear, however, how older and unhealthy individuals might react to such stimulation.

The cold pressor test has been characterized as a passive physiological stressor (30). Such a classification has made it appealing as an experimental stressor, as ideally all subjects should respond alike without confounding psychological factors. Nonetheless, distinctly psychological coping skills and personality variables can shape the arousal profile of the cold pressor. For example, subjective pain during the cold pressor has been shown to correlate with increases in heart rate, and thus, decreases in vagal tone (Reeves, 1982). Subjects reporting pain would thus be expected to differentially experience greater decreases in vagal tone. Subjects in the paced breathing condition reported significantly greater ratings of pain than controls ( $t$ (17) $=2.21, p=0.042$ ). Contrary to past research, this group also exhibited the greatest overall increase in vagal tone, although their performance during the cold pressor tests remains uncertain. Regrettably, baseline pain ratings were not collected for the first cold pressor test (Figure 4).

Turner (30) suggests capitalizing on individual differences in physiological stress reactivity by forming reactor subgroups, such as "High and Low Heart Rate Reactors" or, in the case of this experiment: "Vascular and Cardiac Reactors." Although such an analysis was attempted on the present results, the resulting sample sizes were too small for analysis.

Interestingly, it may be possible to boost the proportion of subjects in either reactor subgroup. Groups such as gender and ethnicity have been shown to differ on these reactivity dimensions, thus providing an avenue by which to select a subject population predisposed to a particular reactor subtype. For example, relative to Caucasian Americans, African Americans tend to respond vascularly to stress induced increases in blood pressure (1). Future research using the cold pressor test should attempt to pre-select subjects prone to cardiac reactivity. 
Figure 4: Subjective pain ratings of the second cold pressor test by group. $*(\mathrm{t}(17)=2.21, \mathrm{p}=0.042)( \pm$ standard error $)$

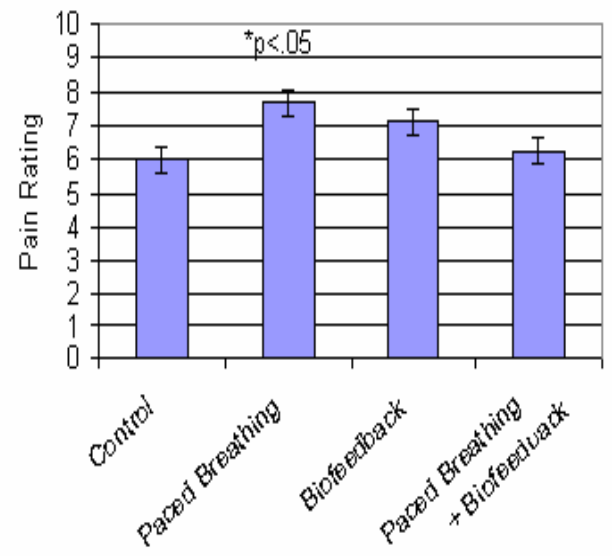

Aiternatve stressors

Although the majority of contemporary stressevoking events are not life threatening as they were during hunter-gatherer times, these challenges nevertheless elicit similar physiological arousal (30). Hence, psychological tasks involving active and continuous mental effort, such as serial subtraction, video games, and speech tasks, have all successfully been used to elicit reliable and stable physiological arousal.

Distinctly cognitive traits, such as locus of control and cynical mistrust, have also been shown to influence cardiac reactivity. Both an internal locus of control and high hostility, as measured by the Cook and Medley (8) hostility scale, can predispose an individual to greater cardiovascular reactivity $(27,29)$. Similarly, Houston's (1992) model postulates a relationship between personality characteristics and cardiac reactivity via emotional and motivational arousal.

In order to mitigate the confounding effects of personality in research paradigms such as the present study, one must make a concerted effort to minimize any situational factors that could trigger a particular personality characteristic. Thus, for example, experimental instructions should be taped and carefully worded to avoid any desirability effects, and minimize any social and personality influences on reactivity. Such issues may have been present in this investigation.

\section{CONCLUSION}

Significant increases in vagal tone were produced using paced respiration, and the combination of paced respiration and biofeedback. However, due to the failure of the cold pressor test to induce consistent and homogeneous cardiac reactivity, and the possible confounding effects of vascular hemodynamic reactivity, this study was unable to address the durability of these increases.

The importance of vagal tone to cardiovascular health, however, is quite evident, especially in those individuals with cardiovascular disease. Evidence to support non-pharmacological methods to increase cardiac vagal activity; on the other hand, has gained only marginal application at the clinical level $(19,28)$. Increasingly, however, a taxed and bewildered medical system is looking to non-conventional approaches to the management of chronic illnesses. Such approaches as Mindfulness Based Stress Reeducation (MBSR) have made great gains in substantiating behavioral and lifestyle modification as essential components of health. Dr. Jon Kabat-Zinn (16) has advocated responding to stress with mindfulness, or the complete acceptance of moment-to-moment events, thoughts, and emotions. Interestingly, MBSR involves much slower and deeper breathing. As previously discussed, ample evidence exists to support such breathing patterns as fostering increased cardiac parasympathetic activity. One need not dismiss slow breathing as efficacious simply because of its simplicity. Rather, the need for a convenient, easy to use, accessible, affordable, and efficacious strategy to increase RSA and vagal tone, makes respiration an attractive technique.

\section{ACKNOWLEDGMENTS}

The author would like to acknowledge the assistance of Michael Alper for kindly accommodating the lab space and time for this experiment as well as Max Lewkowski for his thorough and helpful revisions, advice, and support. In addition, the indispensable advice and support from Ms. Tone Liodden was of great impact to the thought and perseverance underlying the present paper. Furthermore, generous support from Dr. Blaine Ditto during all stages of this project was much appreciated. Both his and Dr. David Ostry's keen comments helped create a cogent and factually accurate paper.

\section{REFERENCES}

1. Anderson, N.B., McNeily, M., and Myers, H. Toward understanding race differences in autonomic reactivity, A proposed contextual model. In J.R. Turner, A. Sherwood, and K.C Light (Eds), Individual differences in cardiovascular response to stress. New York, Plenum, 1992.

2. American Heart Association. Heart Disease and Stroke Statistics -2003 Update. http,//www.americanheart.org/downloadable/ heart/ 1040391091015HDS_Stats_03.pdf 2003.

3. Ashby, W.R. An Introduction to Cybernetic. New York, John Wiley and Sons. Inc, 1963.

4. Benson, H., Beary, J.F, and Carol, M.P. The relaxation response. Psychiatry 37, 37-46; 1974.

5. Bentson, G.G, Cacioppo, J.T., and Quigley, K.S. Respiratory Sinus Arrhythmia, Autonomic origins, physiological 
mechanism, and psychophysiological implications. Psychophysiology 30, 183-196; 1993.

6. Birbaumer, N., and Kimmel, H.D. Biofeedback and selfregulation. New York, Hillsdale, N.J. L. Erlbaum Associates, 1979.

7. Borovikova, L. V., Ivanova S., Zhang M., et al. Vagus nerve stimulation attenuates the systemic inflammatory response to endotoxin. Nature 405, 458-462; 2000.

8. Cookm W.W, and Medley, D.M. Proposed hostility and pharisaic-virtue scales for the MMPI. Journal of Applied Psychology 38, 414-418; 1954.

9. Eckberg. D.L. Parasympathetic cardiac control in human disease, A critical review of methods and results. American Journal of Physiology 239(5), H581-H593;1980.

10. Eckberg DL, Drabinsky M, Braunwald E. Defective cardiac parasympathetic control in patients with heart disease.. New England J Med. 285(16),877-883; 1971.

11. Eckholdt, K., Bodmann, K. H., Pfiefer, B., et al. Sinus Arrhythmia and heart rate in hypertonic disease. Advances in Cardiology 16, 366; 1976.

12. Fouad, F. M., Tanazi, R.C., Rerrario, et al. Assessment of parasympathetic control of heart rate by a non-invasive method. American Journal of Physiology 246, H838-H842; 1984.

13. Grossman, P., Stembler, G., and Meinhardt, E. Paced respiratory sinus arrhythmia as an index of cardiac parasympathetic tone during varying behavioral tasks. Psychophysiology, 27, 404416; 1990.

14. Hayano, J., Yanada, A., Mukai, S., et al. Severity of arteriosclerosis correlates with the respiratory component of heart rate variability. American Heart Journal 121, 1070-1079; 1991.

15. Heart and Stroke Foundation of Canada. The Changing Face of Heart Disease and Stroke in Canada. Ottawa, Canada; 1999.

16. Kabbat-Zinn, J. Full Catastrophe Living. New York, Delta; 1990.

17. Katona, P.G, and Jib, R. Respiratory Sinus Arrhythmia, A noninvasive measure of parasympathetic cardiac control. Journal of Applied Physiology 39, 801-805; 1975.

18. Kleiger, R.E., Miller, P., Bigger, T., et al. Decreased Heart Rate Variability and Its Association with Increased Mortality after acute myocardial infraction. The American Journal of Cardiology 59, 256-261; 1987.
19. Lehrer, P., Smetankin, A., and Potapova, T. Respiratory Sinus Arrhythmia Biofeedback Therapy for Asthma, A Report of 20 Unmedicated Pediatric Cases Using the Smetankin Method. Applied Psychophysiology and Biofeedback 25(3), 193-200; 2000.

20. Lown, B., and Verrier, R.L. Neural activity and ventricular fibrillation. New England Journal of Medicine 364, 1165-1170; 1976.

21. O'Hair, D.E. Biofeedback, Review, History, and Application. http.//free.hostdepartment.com/d/deohair/psychoph.html 1988.

22. Porges, S.W. Respiratory Sinus Arrhythmia, Physiological basis, quantitative methods, and clinical implications. In P. Grossman, K.H. Jameson, \& D. Vaitl. Cardiorespiratory and cardiosomatic psychophysiology. New York, Plenum Pub Corp,101-106; 1986.

23. Porges, S.W., and Vagal Tone, A physiologic marker of stress vulnerability. Pediatrics 90(3), 498-504; 1993.

24. Reeves, J.L., and Shapiro, D. Heart Rate biofeedback and cold pressor pain. Psychophysiology 19(4), 393-403; 1982.

25. Reeves, J.L., and Shapiro, D. Heart Rate Reactivity to the cold pressor stress following biofeedback training. Biofeedback and Self Regulation 8(1), 87-89; 1983.

26. Reyes Del Paso, G., Godoy, J., and Vila, J. Self-Regulation of Respiratory Sinus Arrhythmia. Biofeedback and Self Regulation 17(4), 261-275; 1992.

27. Rotter, J. Generalized expectancies of internal and external control of reinforcement. Psychological Monographs 80 (1), Whole No 609; 1966.

28. Sakakibara, M., Takeuchi, S, and Hayano, J. Effect of relaxation training on cardiac parasympathetic tone. Psychophysiology 31 , 223-228; 1994.

29. Suarez, E.C, and Williams, R.B., Jr. Situational determinants of cardiovascular emotional reactivity in high and low hostile men. Psychosomatic Medicine 51, 414-418; 1989.

30. Turner, J.R. Cardiovascular Reactivity and Stress, Patterns of Physiological Response. New York, Plenum; 1994.

31. Victor, R., Mainardi, A., and Shapiro, D. Effects of Biofeedback and Voluntary Control Procedures on Heart Rate and Perception of Pain during the Cold Pressor Test. Psychosomatic Medicine 40(1), 216-225; 1978.

Dorey Glenn obtained his B.Sc. in Psychology from McGill University. He is currently an MA/MD student at Touro College and the Technion Institute of Technology in Haifa, Israel. He is interested in the use of mindfulness / awareness techniques, such as meditation, for health and well-being. His current career goals include practicing as a rural family physician or geriatrician. 